\title{
KLEIN'S GROUP DEFINES AN EXCEPTIONAL SINGULARITY OF DIMENSION 3
}

\author{
D. MARKUSHEVICH AND YU. G. PROKHOROV
}

\section{INTRODUCTION}

The aim of this paper is to construct examples of canonical exceptional singularities. Canonical (as well as terminal, log terminal and log canonical) singularities appear naturally in the minimal model theory and were studied by Reid, Mori, Kollár, Shokurov and others. Recently Shokurov [Sh1] introduced the notion of exceptional singularity, see Definition 1.2. This notion is closely connected with the inductive approach to the classification of singularities, flips, divisorial contractions, etc. The key ingredient of the inductive approach in its modern setting is the search of complements, that is, of good divisors in the multiple anticanonical systems (see Definition 1.4). In fact the main result of [Sh1] is that two-dimensional complements can be divided into two parts: regular and exceptional. Regular ones occur in 1-, 2-, 3-, 4- or 6-uple anticanonical systems and have a rather simple structure. Exceptional ones can only occur in the neighborhood of an exceptional singularity; they are more complicated to study but they belong, up to birational isomorphisms, to a finite number of families and, at least in principle, can be classified [Sh1]. By using standard arguments with Kawamata-Viehweg vanishing and the inversion of adjunction (see [Ut, 19.6]) these results can be applied to study three-dimensional log canonical singularities and, even more generally, extremal contractions [Sh1, §7].

In dimension 3, Shokurov [Sh1, §7] gave an example of a log Del Pezzo surface with no numerical obstructions to the existence of its blow down to an exceptional canonical singularity, but at the moment it is not clear whether such a singularity really exists. In the present paper, we construct first examples of 3-dimensional canonical exceptional singularities.

1991 Mathematics Subject Classification. 14E30.

The second author was partially supported by the Russian Foundation of Fundamental Research grant 96-01-00820 and by a grant PECO-CEI from the Ministry of Higher Education of France. 
Our examples belong to the class of quotient singularities: they are quotients of $\mathbb{C}^{3}$ by the action of Klein's simple group J of order 168 , and of its central extension $J^{\prime}$ by the 3-rd roots of unity, of order 504 . Remark, that these quotient singularities have been already investigated in relation to another problem in [Mar and [Ro. It was proved, that they and, more generally, any quotient of $\mathbb{C}^{3}$ by a finite subgroup of the special linear group, are crepant, that is, admit resolutions with trivial canonical class. Thus, they are in a sense opposite to terminal, or totally discrepant singularities inside the class of canonical ones. By Example 1.9, the terminal singularities are always nonexceptional, so, we have a reason to start the search of exceptional ones among crepant quotients.

We use the Miller-Blichfeldt-Dickson classification [MBD of finite subgroups of $S L_{3}(\mathbb{C})$, and show, that for some classes of this classification the quotient is not exceptional. These include the reducible, imprimitive groups, the icosahedral group $H$ and its central extension $H^{\prime}$ of order 180. All these groups have a semiinvariant of degree $\leq 3$. It is plausible, that the quotients by the subgroups without semiinvariants of degree $\leq 3$ are exceptional. But the proof of the exceptionality is not so easy as that of the nonexceptionality: in the first case, one has to verify the minimal discrepancies for all possible boundaries, whereas, in the second case, it suffices to find one nonexceptional boundary. We prove the exceptionality of our examples by the rule of contraries, in using the existence of a nonexceptional 1-, 2-, 3-, 4- or 6-complement of the canonical divisor (according to [Sh1]) for a nonexceptional singularity. This implies that it suffices to verify only the boundaries given by the semiinvariants of degree $\leq 18$. The rest of the proof relies heavily upon the classical results of Klein on the group $J$ : enumeration of the orbits, list of invariants, the configuration of the non-free locus of its action on the projective plane.

We will describe now briefly the contents of the article by sections.

Section 1 is preliminary, it contains definitions and some facts for later use. In Section 2 we describe our approach to the prooof of the exceptionality of quotient singularities. In particular we prove (Corollary 2.3) that a quotient singularity $\mathbb{C}^{3} / G$ can be exceptional only if $G$ is primitive. In Section 3 the main result (Theorem 3.1) is proved.

Acknowledgements. The authors would like to thank V. V. Shokurov for useful remarks. 


\section{DEFinitions AND PRELIMINARY RESUlts}

We follow essentially the terminology and notation of [Ut], [Sh] and Sh1] (see also [Ko for a nice introduction to the subject).

Definition 1.1. Let $(X \ni P)$ be a normal singularity (not necessarily isolated) and let $D=\sum d_{i} D_{i}$ be a divisor on $X$ with real coefficients. $D$ is called a boundary if $0 \leq d_{i} \leq 1$ for all $i$. It is called a subboundary, if it is majorated by a boundary. A proper birational morphism $f: Y \longrightarrow X$ is called a $\log$ resolution of $(X, D)$ at $P$, if $Y$ is nonsingular near $f^{-1}(P)$ and $\operatorname{Supp}(D) \cup E$ is a normal crossing divisor on $Y$ near $f^{-1}(P)$, where $D$ is used to denote both the subboundary on $X$ and its proper transform on $Y$, and $E=\cup E_{i}$ is the exceptional divisor of $f$. The pair $(X, D)$ or, by abuse of language, the divisor $K_{X}+D$ is called terminal, canonical, Kawamata log terminal (klt), purely log terminal (plt), and, respectively, log canonical (lc) near $P$, if the following conditions are verified:

(i) $K_{X}+D$ is $\mathbb{R}$-Cartier.

(ii) Let us write for any proper birational morphism $f: Y \longrightarrow X$

$$
K_{Y} \equiv f^{*}\left(K_{X}+D\right)+\sum a(E, X, D) E,
$$

where $E$ runs over prime divisors on $Y, a(E, X, D) \in \mathbb{R}$, and $a\left(D_{i}, X, D\right)=$ $-d_{i}$ for each component $D_{i}$ of $D$. Then, for some log resolution of $(X, D)$ at $P$ and for all prime divisors $E$ on $Y$ near $P$, we have: $a(E, X, D)>0$ (for terminal), $a(E, X, D) \geq 0$ (for canonical), $a(E, X, D)>$ -1 and no $d_{i}=1$ (for klt), $a(E, X, D)>-1$ (for plt, without any restriction on the subboundary $D)$, and, respectively, $a(E, X, D) \geq-1$ (for lc).

The coefficients $a(E, X, D)$ are called discrepancies of $f$, or of $(X, D)$; they depend only on the discrete valuations of the function field of $X$ associated to the prime divisors $E$, and not on the choice of $f$. We will identify prime divisors with corresponding discrete valuations, when speaking about 'divisors $E$ over $X$ ' without indicating, on which birational model $E$ is realized. The conditions given by inequalities in part (ii) of the above definition do not depend on the choice of a log resolution. The lc (as well as terminal, canonical, klt, plt) condition is obviously monotonic on $D$ : if $(X, D)$ is lc at $P$, then $\left(X, D^{\prime}\right)$ is also lc at $P$ for any $D^{\prime} \leq D$ such that $K_{X}+D^{\prime}$ is $\mathbb{R}$-Cartier. Thus, for any boundary $D$, which is a $\mathbb{R}$-Cartier divisor on a $\mathbb{Q}$-Gorenstein variety we can define the $\log$ canonical threshold of $(X, D)$ at $P$ :

$$
c_{P}(X, D)=\max \{\alpha \in \mathbb{R} \mid(X, \alpha D) \text { is } \log \text { canonical }\}
$$


Definition 1.2 ([Sh1, 1.5]). Let $(X \ni P)$ be a normal singularity and let $D=\sum d_{i} D_{i}$ be a boundary on $X$ such that $K_{X}+D$ is $\log$ canonical. The pair $(X, D)$ is said to be exceptional if there exists at most one exceptional divisor $E$ over $X$ with discrepancy $a(E, X, D)=-1$. The singularity $(X, P)$ is said to be exceptional if $(X, D)$ is exceptional for any $D$ whenewer $K_{X}+D$ is $\log$ canonical.

Remark 1.3. By the connectedness result [ST, 5.7], [Ut, 17.4], the set of divisors with discrepancies $\leq-1$ in $Y$ for any proper birational morphism $f: Y \longrightarrow X$ is connected. Therefore, if a log canonical pair $(X, D)$ is nonexceptional, then there exist infinitely many divisors with discrepancy -1 , which can be constructed by blowing up the compoments of intersections of pairs of such divisors.

Definition $1.4([S h, 5.1])$. Let $(X, P)$ be a normal singularity, $D=$ $S+B$ a subboundary, such that $B, S$ have no common components, $S$ is a reduced divisor, and $B=\sum b_{i} B_{i}$ with all $b_{i}<1$, that is, $\lfloor B\rfloor=0$, where $\lfloor\cdot\rfloor$ denotes the integer part. Then one sais that $K_{X}+D$ is $n$ complemented, if there exists a $\mathbb{Q}$-divisor $D^{+}$, such that the following conditions are verified:

(i) $n D^{+}$has integer coefficients ;

(ii) $n D^{+} \sim-n K_{X}$;

(iii) $n D^{+}-n S-\lfloor(n+1) B\rfloor \geq 0$;

(iv) $K_{X}+D^{+}$is lc.

The divisor $K_{X}+D^{+}$is called a $n$-complement of $K_{X}+D$. Remark, that if $D$ is a boundary, then so is $D^{+}$.

Example 1.5 ([Sh, 5.2.3, 5.6], [Sh1, 1.5]). Let $(X, P)$ be a two-dimensional quotient singularity. Then the following conditions are equivalent:

(i) $(X \ni P)$ is exceptional,

(ii) $(X \ni P)$ is of type $E_{6}, E_{7}$ or $E_{8}$ (in the generalized sense of [1], $[\mathrm{Br}]$ ); this means that the dual (weighted) graph of the minimal resolution of such a singularity has a single 3 -valent vertex $\stackrel{-b}{\circ}$, $b \geq 2$ with three chains issued from it, exactly one of them being of type $-{ }^{-2}$ (see [B], [1], or [Ut, Ch. 3] for a more precise description),

(iii) there are no 1- or 2-complements $K_{X}+D$ such that $P \in \operatorname{Supp}(D)$ (but there is a 3-, 4- or 6-complement),

(iv) $(X \ni P)$ is analytically isomorphic to a quotient $\mathbb{C}^{2} / G$, where $G$ is a finite subgroup of $G L_{3}(\mathbb{C})$ without reflections of dihedral, tetrahedral or icosahedral type $[\mathrm{Br}]$. This means that the image of $G$ 
in $P G L_{2}(\mathbb{C}) \simeq S O_{3}(\mathbb{C})$ is the dihedral, tetrahedral or icosahedral group in the usual sense.

The following theorem is a consequence of the proof of Shokurov's theorem Sh1, 7.1].

Theorem 1.6. Let $(X \ni P)$ be a nonexceptional three-dimensional log canonical singularity. Then $K_{X}$ is either 1-, 2-, 3-, 4- or 6-complemented. Moreover, there exists such a nonexceptional complement $K_{X}+D$.

Lemma 1.7. Assume that there exists a reduced divisor $S=\sum S_{i}$ passing through $P$ such that $K_{X}+S$ is log canonical. Then $(X \ni P)$ is nonexceptional.

Proof. Take a general hyperplane section through $P$. Then, for some $0 \leq \alpha \leq 1$, the $\log$ divisor $K_{X}+S+\alpha H$ is $\log$ canonical, but not purely log terminal. By Remark 1.3, the set of divisors with discrepancy $a(, X, S+\alpha H)=-1$ on a resolution $Y \rightarrow X$ is connected, and by construction, we have at least two of them, one coming from $S$ and the other one, say $E$, exceptional over $X$. So we can get infinitely many divisors with discrepancy -1 in blowing up the curves of intersections.

Example 1.8. By definition and Lemma 1.7 any three-dimensional cDV-singularity is nonexceptional.

Example 1.9. Let $(X \ni P)$ be a three-dimensional terminal singularity and $S \in\left|-K_{X}\right|$ a general element (in the Gorenstein case, we should additionally suppose that $S \ni P)$. By [R, 6.4] and the inversion of adjunction [Sh, 3.3, 3.12, 5.13], [Ut, 17.6] $K_{X}+S$ is purely log terminal (and even canonical). Therefore, all terminal singularities are nonexceptional. Of course, these arguments use the classification of terminal singularities. Shokurov (cf. [R, 6.5]) posed the problem to prove this fact directly.

\section{Quotient Singularities}

Now let $(X \ni P)$ be a three-dimensional quotient singularity, i. e. $(X \ni P)=\left(\mathbb{C}^{3} \ni 0\right) / G$, where $G \subset G L_{3}(\mathbb{C})$ is a finite subgroup. We may assume that $G$ contains no quasi-reflections, for if $G$ contains quasi-reflections, then there exists another subgroup $G^{\prime}$ of $G L_{3}(\mathbb{C})$, which does not contain a quasi-reflection and such that $\mathbb{C}\left[y_{1}, y_{2}, y_{3}\right]^{G} \simeq$ $\mathbb{C}\left[y_{1}, y_{2}, y_{3}\right]^{G^{\prime}}$ as $\mathbb{C}$-algebras. 
Let $\pi: V \rightarrow X$ be the quotient morphism, where $V=\mathbb{C}^{3}$. Let $D$ be a boundary on $X$ and let $D^{\prime}:=\pi^{*} D$. By [Sh, 2.2], [Ut, 20.3], [Ko, ] $K_{X}+D$ is $\log$ canonical (resp., plt, klt) iff so is $K_{V}+D^{\prime}$.

Lemma 2.1. In the above notation, $(X, D)$ is exceptional iff $\left(V, D^{\prime}\right)$ is.

Proof. Assume that $\left(V, D^{\prime}\right)$ is exceptional. Then by [Sh2, 3.1], [0t, 17.10] there exists a blow-up $g: W \rightarrow V$ such that $K_{W}+S+B^{\prime}=$ $g^{*}\left(K_{V}+D^{\prime}\right)$ is purely $\log$ terminal, where $S$ is the (irreducible) exceptional divisor of $g$ and $B^{\prime}$ is the proper transform of $D^{\prime}$. This blow-up is unique up to isomorphism, because $g$ is projective and $\rho(W / V)=1$ [Ut, 6.2]. So we can define an action of $G$ on $W$ making $g$ equivariant. Let $\varphi: W \rightarrow Y$ be the quotient morphism and put $E:=\varphi(S)$ and $B:=\varphi\left(B^{\prime}\right)$. We have the following commutative diagram

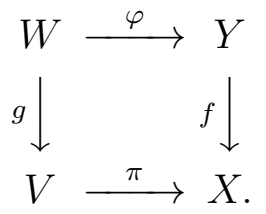

By the ramification formula

$$
K_{W}+S+B^{\prime}=\varphi^{*}\left(K_{Y}+E+B\right),
$$

whence

$$
K_{Y}+E+B=f^{*}\left(K_{X}+D\right) .
$$

By [Sh, 2.2], [णt, 20.3] $K_{Y}+E+B$ is purely log terminal. Therefore, $K_{X}+D$ is exceptional.

Conversely, assume that $K_{X}+D$ is exceptional. Let $W$ be the normalization of $V \times_{X} Y$. We again have the diagram (1) and relations (2), (3). Similarly, by [Sh, 2.2], [0t, 20.3], $K_{W}+S+B^{\prime}$ is purely log terminal, hence $K_{V}+D^{\prime}$ is exceptional.

Lemma 2.2. In the notation of Lemma 2.1, assume that $G$ has a semiinvariant of degree $\leq 3$. Then $(X \ni P)$ is nonexceptional.

Proof. Let $\psi$ be such a semiinvariant of minimal degree $d \leq 3$, let $D^{\prime}$ be its zero locus, and let $D:=\pi\left(D^{\prime}\right)_{\text {red }}$. Then $D^{\prime}=\pi^{*}(D)$. By Lemma 1.7 it is sufficient to show that $K_{V}+D^{\prime}$ is $\log$ canonical. Note that $\psi$ is homogeneous, so $D^{\prime}$ is a cone over a plane curve $C$ of degree $d \leq 3$. If $C$ is nonsingular, then $D^{\prime}$ has a unique singular point which can be resolved by only one blow-up with $a\left(\nu, V, D^{\prime}\right)=2-$ mult $_{0} \psi \geq-1$, so $K_{V}+D^{\prime}$ is $\log$ canonical in this case. If $C$ has a unique singular point, then $G$ has an eigenvector, say $v \in \mathbb{C}^{3}$. But then $G$ has an invariant 
plane, orthogonal to $v$ and we can take $d=1$ and $C$ is nonsingular. The same arguments work if $C$ has exactly two singular points. In the remaining case $C$ is the union of three lines in general position. Then $\left(V, D^{\prime}\right)$ is already log nonsingular (that is, may be taken as its own log resolution), and so $K_{V}+D^{\prime}$ is log canonical.

The subgroup $G \in G L_{3}(\mathbb{C})$ is said to be reducible, if it has a proper invariant subspace in $\mathbb{C}^{3}$. $G$ is called imprimitive, if there exists a triple of lines $L_{1}, L_{2}, L_{3}$ in $\mathbb{C}^{3}$, permuted by $G$ via a representation $G \longrightarrow S_{3}$, where $S_{n}$ denotes the symmetric group of permutations on $n$ elements.

Corollary 2.3. If $(X \ni P)$ is exceptional, then $G$ is irreducible and primitive.

Proof. If $G$ is reducible, it has a semiinvariant of degree 1 . If it is imprimitive, it has a semiinvariant of degree 3 , defining the union of three planes spanned by pairs of lines from $L_{1}, L_{2}, L_{3}$. The result follows by Lemma 2.2.

The finite subgroups of $G \subset G L_{3}(\mathbb{C})$ were classified by MillerBlichfeldt-Dickson MBD modulo extension by certain scalar matrices (compare with $[\mathbb{P}]$ ). There are 9 types of such groups, denoted by A,B, $\ldots, \mathrm{J}$ in $\mathrm{MBD}$. As soon as we are looking for those which yield exceptional quotient singularities, we have to test only primitive irreducible ones. They belong to the 6 types E, F, G, H, I, J. The orders of the associated collineation groups $P G=G /\left(G \cap \mathbb{C}^{*}\right) \subset P G L_{3}(\mathbb{C})$ are 36, $72,216,60,360,168$; the first three are solvable, and the last three are simple. The collineation groups from $\mathrm{G}$ to $\mathrm{J}$ have their names: the Hessian group, the icosahedral one, the alternating group of degree 6, and, finally, Klein's simple group.

Proposition 2.4. The quotients of $\mathbb{C}^{3}$ by the subgroups of $S L_{3}(\mathbb{C})$ of type $H$ are nonexceptional.

Proof. There are two such groups (see, e. g., [P] or [Ro]): the icosahedral group $G$ of order 60, and its central extension $G^{\prime}$ of order 180 . The invariants of $G$ are well-known; see, for example, MBD, Sect. 116]. There is an invariant of degree 2, which is a semiinvariant of $G^{\prime}$. (This also follows from the fact that $G$ is a subgroup of $S L_{3}(\mathbb{R})$ and therefore has an invariant quadratic form $[\mathrm{Sp}, 4.2 .15])$. The result follows by Lemma 2.2.

2.5. Now we will explain the logic of our approach to the proof of the exceptionality of a quotient singularity, which will be applied in the next section to Klein's group. Assume that $(X \ni P)$ is nonexceptional. By Theorem 1.6 there exists a nonexceptional log canonical $K_{X}+D$ 
such that $n\left(K_{X}+D\right) \sim 0$ for $n \in\{1,2,3,4,6\}$. Further, we will use notations of Lemma 2.1. The integer divisor $F:=n D^{\prime}$ locally near 0 can be defined by a semiinvariant function, say $\psi$.

Moreover $n D \sim-n K_{X}$ iff the form $\psi\left(d x_{1} \wedge d x_{2} \wedge d x_{3}\right)^{-n}$ is invariant, i. e.

$$
g(\psi)=\operatorname{det}(g)^{n} \psi \quad \text { for all } \quad g \in G .
$$

Denote $d:=\operatorname{mult}_{0}(\psi)$. Let $\sigma: W \rightarrow V=\mathbb{C}^{3}$ be the blow-up of the origin and let $S \simeq \mathbb{P}^{2}$ be the exceptional divisor. Then $K_{W}=$ $\sigma^{*} K_{V}+2 S$ and $\sigma^{*} F=R+d S$, where $R$ is the proper transform of $F$. Therefore the discrepancy of $S$ is

$$
a\left(S, V, D^{\prime}\right)=2-\frac{1}{n} \operatorname{mult}_{0}(\psi) \geq-1 .
$$

So we have $d=\operatorname{mult}_{0}(\psi) \leq 3 n \leq 18$.

Further

$$
K_{W}+S+\frac{3}{d} R=\sigma^{*}\left(K_{V}+\frac{3}{d} F\right) .
$$

By [Ka, Lemma 3.10] $K_{V}+\frac{3}{d} F$ is $\log$ canonical iff so is $K_{W}+S+\frac{3}{d} R$. In this case the pair $(V, \alpha F)$ is exceptional for all $0 \leq \alpha \leq \frac{3}{d}$ iff $K_{W}+$ $S+\frac{3}{d} R$ is purely $\log$ terminal; by the above, we need this assertion only for $\alpha=\frac{1}{n}$ with $n \in\{1,2,3,4,6\}$, but we will have a stronger property with $\alpha$ not necessarily of this form. The plt condition for $K_{W}+S+\frac{3}{d} R$ is equivalent to that $K_{S}+\frac{3}{d} C$ is Kawamata log terminal, where $C=R \cap S$ [Sh, 5.13], [0t, 17.6]. It is clear that $C$ is given by the equation $\psi_{\min }=0$, where $\psi_{\min }$ is the homogeneous component of $\psi$ of minimal degree $d$. Therefore we have

Proposition 2.6. In the above notations, if $K_{S}+\frac{3}{d} C$ is Kawamata log terminal, then $(V, \alpha F)$ is exceptional for any $0 \leq \alpha \leq \frac{3}{d}$.

Remark 2.7. If $K_{S}+\frac{3}{d} C$ is $\log$ canonical, but not Kawamata log terminal, then $(V, F)$ is nonexceptional. If $K_{S}+\frac{3}{d} C$ is not log canonical, then we can conclude nothing (possibly in this situation we have to consider some weighted blow-up).

Lemma 2.8. Notations as above. Assume that $K_{S}+\frac{3}{d} C$ is not Kawamata $\log$ terminal and $C$ is a singular irreducible curve. Then there exists an orbit of $G$ consisting of at most 10 singular points of $C$.

Proof. Let $P \in C$ be a singular point of maximal multiplicity $m$ and let $r$ be the number of points in the orbit $G \cdot P$. Denote by $c=c(S, C)$ the (global) $\log$ canonical threshold of $(S, C)$. By our assumption $c \leq 3 / d$. 
On the other hand, $c \geq 1 / m \sqrt{\mathrm{Kg}}$, Lemma 8.10]. This gives us $d \leq 3 m$. Let $g$ be the genus of the normalization of $C$. Then

$$
0 \leq g \leq \frac{(d-1)(d-2)}{2}-r \frac{m(m-1)}{2} .
$$

Taking into account that $d \leq 3 m$, we obtain

$$
-2 \leq 2 g-2 \leq(9-r) m(m-1)
$$

This implies the assertion.

\section{KLEIN'S GROUP}

The aim of this section is to prove the exceptionality of the quotients of $\mathbb{C}^{3}$ by the subgroups of $S L_{3}(\mathbb{C})$ of type $\mathrm{J}$ in the classification of [MBD. There are two such groups, see $[\mathbb{P}]$ or $[\mathbb{R O}]$ : Klein's simple group $J_{168}$ of order 168 and its central extension $J_{504}^{\prime}$ of order 504 .

Theorem 3.1. Let $G \subset S L_{3}(\mathbb{C})$ be $J_{168}$ or $J_{504}^{\prime}$. Then the singularity of the quotient $\mathbb{C}^{3} / G$ at the origin is exceptional.

We will briefly describe the irreducible representation of $J_{168}$ in $\mathbb{C}^{3}$ following $[\mathrm{W}]$ and $[\mathbb{K}]$. Another description of $J_{168}$ see in $[\mathrm{Sp}$, Sect. 4.6]. Let $y_{1}, y_{2}, y_{3}$ be coordinates in $\mathbb{C}^{3}$. The group $J_{168}$ is generated by 3 elements $\tau, \chi, \omega$ of orders $7,3,2$ respectively, and the representation is defined by

$$
\begin{aligned}
& \tau=\left(\begin{array}{ccc}
\epsilon & & 0 \\
& \epsilon^{2} & \\
0 & & \epsilon^{4}
\end{array}\right) \quad, \quad \epsilon=\exp \left(\frac{2 \pi i}{7}\right), \\
& \chi=\left(\begin{array}{ccc}
0 & 0 & 1 \\
1 & 0 & 0 \\
0 & 1 & 0
\end{array}\right) \quad, \quad \omega=\left(\begin{array}{ccc}
\alpha & \beta & \gamma \\
\beta & \gamma & \alpha \\
\gamma & \alpha & \beta
\end{array}\right) \text {, } \\
& \alpha=-\frac{2 \sin \frac{8 \pi}{7}}{\sqrt{7}}, \quad \beta=-\frac{2 \sin \frac{4 \pi}{7}}{\sqrt{7}}, \quad \gamma=-\frac{2 \sin \frac{2 \pi}{7}}{\sqrt{7}},
\end{aligned}
$$

The second group $J_{504}^{\prime}$ is generated by $J_{168}$ and the scalar matrix with $\exp \frac{2 \pi i}{3}$ on the diagonal.

Now, we will describe the semiinvariants of these groups. First of all, since $J_{168}$ is simple, all its semiinvariants are indeed invariants. They are also semiinvariants of $J_{504}^{\prime}$. According to [ [K] (see also [W]), the algebra of invariants $A=\mathbb{C}\left[y_{1}, y_{2}, y_{3}\right]^{J_{168}}$ is generated by four homogeneous polynomials $f, \Delta, \mathcal{C}, \mathcal{K}$ of degrees $4,6,14,21$ respectively, with one basic relation between them: 


$$
\begin{gathered}
f=y_{1}^{3} y_{3}+y_{2}^{3} y_{1}+y_{3}^{3} y_{2}, \\
\Delta=\frac{1}{54} \operatorname{Hess}(f), \\
\mathcal{C}=\frac{1}{9}\left|\begin{array}{cccc}
f_{y_{1} y_{1}}^{\prime \prime} & f_{y_{1} y_{2}}^{\prime \prime} & f_{y_{1} y_{3}}^{\prime \prime} & \Delta_{y_{1}}^{\prime} \\
f_{y_{2} y_{1}} & f_{y_{2} y_{2}}^{\prime \prime} & f_{y_{2} y_{3}}^{\prime \prime} & \Delta_{y_{2}}^{\prime} \\
f_{y_{3} y_{1}} & f_{y_{3} y_{2}}^{\prime \prime} & f_{y_{3} y_{3}}^{\prime \prime} & \Delta_{y_{3}}^{\prime} \\
\Delta_{y_{1}}^{\prime} & \Delta_{y_{2}}^{\prime} & \Delta_{y_{3}}^{\prime} & 0
\end{array}\right| \\
\mathcal{K}=\frac{1}{14}\left|\begin{array}{ccc}
f_{y_{1}}^{\prime} & \Delta_{y_{1}}^{\prime} & \mathcal{C}_{y_{1}}^{\prime} \\
f_{y_{2}}^{\prime} & \Delta_{y_{2}}^{\prime} & \mathcal{C}_{y_{2}}^{\prime} \\
f_{y_{3}}^{\prime} & \Delta_{y_{3}}^{\prime} & \mathcal{C}_{y_{3}}^{\prime}
\end{array}\right| \\
\mathcal{K}^{2}=\mathcal{C}^{3}+1728 \Delta^{7}+1008 \mathcal{C} \Delta^{4} f-88 \mathcal{C}^{2} \Delta f^{2}-60032 \Delta^{5} f^{3} \\
+1088 \mathcal{C} \Delta^{2} f^{4}+22016 \Delta^{3} f^{6}-256 \mathcal{C} f^{7}-2048 \Delta f^{9} .
\end{gathered}
$$

In the proof of the Theorem, we will follow the logic scheme outlined in Subsect. 2.5. In the notations of 2.5 let $F$ be given by the equation $\psi=0$, where $\psi=\sum a_{i j k l} f^{i} \Delta^{j} \mathcal{C}^{k} \mathcal{K}^{l} \quad(i \geq 0, j \geq 0, k \geq 0, l=0$ or 1 , such that the the lowest degree of non-zero terms $d=\operatorname{mult}_{0} \psi=$ $\operatorname{deg} \psi_{\min } \leq 18$. For $G=J_{168}$, up to a constant factor of proportionality, $\psi_{\min }$ can be only one of the following functions:

$f, \Delta, f^{2}, f \Delta, \lambda f^{3}+\mu \Delta^{2}, \lambda f^{2} \Delta+\mu \mathcal{C}, \lambda f^{4}+\mu \Delta^{2} f, \lambda \Delta^{3}+\mu f^{3} \Delta+\nu f \mathcal{C}$,

where $\lambda, \mu, \nu$ are arbitrary complex constants. The list of initial forms for $G=J_{504}^{\prime}$ is even shorter: according to (4), the character of $\psi$ is equal to $g \mapsto(\operatorname{det} g)^{n}$, hence it is trivial, because $G \subset S L_{3}(\mathbb{C})$. So, only polynomials of degree divisible by 3 should be kept in the case of $J_{504}^{\prime}$. The rest of the proof depends only on the pair $(S, C)=\left(\mathbb{P}^{2},\left(\psi_{\min }\right)\right)$, and is done simultaneously for the two groups.

Case 1. $\psi_{\min }=f^{k}$ or $\Delta^{k} ; \operatorname{deg} \psi_{\min }=4 k$ or, resp. $6 k$. The reduced curve $C_{\text {red }}$ is nonsingular, so the pair $\left(S, C_{\text {red }}\right)$ is $\log$ nonsingular. Hence $\left(S, \alpha C_{\text {red }}\right)$ is klt for any $\alpha<1$. Hence $\left(S, \frac{3}{d} C\right)=\left(S, \frac{3 k}{d} C_{\text {red }}\right)$ is klt. By Proposition 2.6, the pair $(V, \alpha F)$ is exceptional for any $0 \leq \alpha \leq \frac{3}{d}$, and we are done.

Case 2. $\psi_{\min }=f^{i} \Delta^{j}, 10 \leq d=4 i+6 j \leq 18$. Here $C_{\text {red }}=\{f \Delta=0\}$ is singular only at the points of intersection of Klein's quartic $C_{1}=\{f=$ $0\}$ and its Hessian curve $C_{2}=\{\Delta=0\}$, that is at the inflection points of Klein's curve. It is known that they form one orbit of length 24 under the action of $J_{168}$ with representative $(1: 0: 0)$. Hence they are 
ordinary double points of $C_{\text {red }}$, and thus, $\left(S, C_{\text {red }}\right)$ is log nonsingular. Hence $\left(S, \alpha C_{1}+\beta C_{2}\right)$ is klt for any $\alpha<1, \beta<1$. Hence $\left(S, \frac{3}{d}\left(i C_{1}+\right.\right.$ $\left.j C_{2}\right)$ ) is klt, and we are done.

Cases 1 and 2 cover all the invariant curves of degree $<12$, as well as multiples of $C_{1}$ and $C_{2}$.

Case 3. $C$ is a reduced irreducible curve of degree $d \geq 12$. If it is nonsingular, we are done, because $(S, C)$ is log nonsingular, and $\frac{3}{d}<1$. Assume that $\left(S, \frac{3}{d} C\right)$ is not klt. Then $C$ cannot be singular by Lemma 2.8 , because $J_{168}$ does not have orbits in $\mathbb{P}^{2}$ of length $\leq 10$. For the orbits of Klein's group, see, e. g. [W], Sect. 120]; the possible lengths are $21,24,28,42,56,84,168$. Hence $C$ is nonsingular, and this is a contradiction. Hence $\left(S, \frac{3}{d} C\right)$ is always klt in this case, and we are done.

Case 4. $C$ is reducible or non-reduced of degree $12 \leq d \leq 18$. The irreducible components of $C$ are permuted by the action of $J_{168}$. The length of the orbit (if not 1 , for an invariant component), is $\geq 7$, for $J_{168}$ has no non-trivial homomorphisms to symmetric groups $S_{p}$ with $p<7$. Moreover, there are no straight lines as irreducible components, because the action on the dual projective plane of lines in $\mathbb{P}^{2}$ is the same as on $\mathbb{P}^{2}$ itself, and hence the minimal orbit length is 21 (there is indeed an invariant of degree 21 which factors into the product of linear forms, namely, $\mathcal{K}$ ). So, discarding the multiples or combinations of $C_{1}$ and $C_{2}$ covered by Cases 1 and 2, we have three subcases: A. $f \vee \psi_{\min }$ and $\Delta \backslash \psi_{\min }$. Then $d=14,16$, or 18 , and $C$ is the union of $d / 2$ conics. B. $f \mid \psi_{\min }$. Then $d=16$ or 18 , and $\psi_{\min }=f\left(\lambda f^{3}+\mu \Delta^{2}\right)$ with $\lambda \mu \neq 0$ or, resp., $\psi_{\min }=f\left(\lambda f^{2} \Delta+\mu C\right)$ with $\mu \neq 0$. C. $\Delta \mid \psi_{\text {min }}$. Then $\psi_{\min }=\Delta\left(\lambda f^{3}+\mu \Delta^{2}\right)$ with $\lambda \mu \neq 0$.

Subcase $A . d=18$ can be eliminated, because 9 \168. If $d=16$, then the stabilizer of any conic component $\Gamma$ of $C$ is of order 21. Hence, the orbit of the generic point of $\Gamma$ is of length 21 , which is impossible, because there is only one orbit of length 21 in $\mathbb{P}^{2}$ : 'die achtzählige Pole' in the classical terminology. A similar argument shows that $d=14$ is also impossible.

Subcase $B$. If $d=16$, then $C=C_{1} \cup C^{\prime}$, where $C_{1}$ is Klein's curve, and $C^{\prime}=\left\{\lambda f^{3}+\mu \Delta^{2}=0\right\}$ is an irreducible curve of degree 12 . The 24 points $Q_{i}$ of $C_{1} \cap C^{\prime}$ are ordinary cusps of $C^{\prime}$, and the local indices $\left(C_{1}\right.$. $\left.C^{\prime}\right)_{Q_{i}}=2$. By Bezout Theorem, these are the only intersection points. Assume that there is a singular point $R \in C^{\prime}$, different from $Q_{i}$. Let $\delta$ be the length of the orbit of $R$, and $m^{\prime}$ its multiplicity. Then $p_{a}\left(C^{\prime}\right)=$ 
$55 \geq 24+\delta \frac{m^{\prime}\left(m^{\prime}-1\right)}{2}$. Taking into the account the possible lengths of orbits, and the uniqueness of orbits of any length $<84$, we obtain two possible values $\delta=21$ or 28 , and $m^{\prime}=2$. (Remark, that $\delta=28$ really occurs for the dual of Klein's curve, and the corresponding singular points are ordinary double). So, the maximal multiplicity of singular points of $C$ is $m=3$, attained at the points $Q_{i}$. By $[\mathrm{Ko}$, Lemma 8.10], we have the following estimate for the log canonical threshhold: $c_{Q}(S, C) \geq \frac{1}{\operatorname{mult}_{Q} C} \geq \frac{1}{3}$. As $\frac{3}{d}<\frac{1}{3}$ in our case, we are done.

If $d=18$, then $C=C_{1} \cup C^{\prime \prime}$, where $C^{\prime \prime}=\left\{\lambda f^{2} \Delta+\mu C=0\right\}$ is irreducible of degree 14. Similarly to the above, we see that the only possibility for the intersection locus $C_{1} \cap C^{\prime \prime}$ is the orbit of the point $\left\{y_{1}=\exp \frac{2 \pi i}{3} y_{2}=\exp \frac{4 \pi i}{3} y_{3}\right\}$ of length 56 (the other candidate, the orbit of lenght 28 taken with multiplicity 2 , is eliminated because it is not contained in $C_{1}$ !). So, the intersections are transversal. Assuming that there is an extra singular point of $C$, we find at once that the length of its orbit should be 21, and in this case the genus of $C^{\prime \prime}$ is 1 . Klein's group cannot act non-trivially on an elliptic curve. So, $C$ has only 56 ordinary double points as singularities, and hence $(S, \alpha C)$ is klt for all $\alpha<1$.

Subcase $C . C=C_{2} \cup C^{\prime}$, where $C_{2}=\{\Delta=0\}$ is the Hessian curve, and $C^{\prime}=\left\{\lambda f^{3}+\mu \Delta^{2}=0\right\}$ is the irreducible curve of degree 12 from Subcase B. The 24 points $Q_{i}$ are the points of triple intersection of $C_{2}, C^{\prime}$, so by Bezout, there are no other points of intersection. By the argument of Subcase B, $C^{\prime}$ cannot acquire singularities, worse than double points. So, the points $Q_{i}$ are the singular points of $C$ of maximal multiplicity $m=3$. The same argument as in Subcase B ends the proof.

The cases 1-3 cover all possible invariant curves of degree $\leq 18$, and in all these cases the pair $(V, \alpha F)$ is exceptional as soon as it is log canonical. By 2.5, this ends the proof of the Theorem.

Remark 3.2. The exceptional divisor $E$ of $f: Y \rightarrow X=\mathbb{C}^{3} / G$ of Sect. 2 (see (11)) for Klein's group $G$ is the weighted projective plane $\mathbb{P}(4,6,14)=\mathbb{P}^{2} / G$. The different $\operatorname{Diff}_{E}(0)$ (see [Sh1, Ch. 1] for the definition) in this case is $(1 / 2) \Gamma$, where $\Gamma$ is an irreducible curve, the image of 21 lines of fixed points of the elements of order 2 in $G$. The curve $\Gamma$ is given by the equation

$$
\begin{aligned}
0=\mathcal{C}^{3}+ & 1728 \Delta^{7}+1008 \mathcal{C} \Delta^{4} f-88 \mathcal{C}^{2} \Delta f^{2}-60032 \Delta^{5} f^{3} \\
+ & 1088 \mathcal{C} \Delta^{2} f^{4}+22016 \Delta^{3} f^{6}-256 \mathcal{C} f^{7}-2048 \Delta f^{9}
\end{aligned}
$$

of weighted degree 42 (see (6)). It is clear that $\left(E, \operatorname{Diff}_{E}(0)\right)$ is a log Del Pezzo surface. The weighted projective plane $E=\mathbb{P}(4,6,14)$ has 
three singular points of types $A_{1}, A_{2}$ and $\mathbb{C}^{2} / \mathbb{Z}_{7}(2,3)$. The curve $\Gamma$ passes through the first of them and has two singular points: a simple cusp and a tacnode point (lying in the non singular part of $E$ ). It is easy to compute that $K_{E}+(1 / 2) \Gamma$ is $1 / 7-\log$ terminal, so Shokurov's invariant $\delta$ (see [Sh1]) is 0 in our case. A very interesting question is to compute the minimal complement of $K_{E}+\operatorname{Diff}_{E}(0)$.

\section{REFERENCES}

[Br] Brieskorn E. Rationale Singularitäten komplexer Flächen, Invent. Math. 4 (1968), 336-358.

[1] I Iliev A. : Log-terminal singularities of algebraic surfaces. Vestnik Moskov. Univ. Ser. I Mat. Mekh. (1986) No. 3, 38-43; English transl.: Mosc. Univ. Math. Bull. 41 (1986), 46-53

[Kl] Klein, F.: Gesammelte Mathematische Abhandlungen, II. Springer: Berlin, 1973.

[Mar] Markushevich, D.: Resolution of $\mathbb{C}^{3} / H_{168}$, Math. Ann. 308 (1997), 279-289.

[MBD] Miller, G. A., Blichfeldt, H. F., Dickson, L. E.: Theory and Applications of finite groups. Dover Publ. Inc.: New York, 1916.

[P] Pouyanne, N.: Une résolution en singularités toriques simpliciales des singularités-quotient de dimension trois, Ann. Fac. Sci. Toulouse, VI, Ser. Math. 1(1992), 363-398.

[R] Reid M. Young persons guide to canonical singularities, in "Algebraic Geometry, Bowdoin, 1985, Proc. Symp. Pure Math. vol. 46, 1987, pp. 345-414.

[Ro] Roan, S. S.: Minimal resolutions of Gorenstein orbifolds in dimension three, Topology 35(1996), 489-508.

[Ut] Kollár J. et al. Flips and abundance for algebraic threefolds, A summer seminar at the University of Utah, Salt Lake City, 1991. Astérisque. 211 (1992)

[Ko] Kollár J. Singularities of pairs, e-print alg-geom/9601026.

[Sh] Shokurov V. V. 3-fold log flips, Izv. AN SSSR Ser. mat. 56 (1992), 105-201 \& 57 (1993), 141-175; English transl. Russian Acad. Sci. Izv. Math. 40 (1993), 93-202 \& 43 (1994), 527-558

[Sh1] Shokurov V. V. Complements on surfaces, preprint, (1997)

[Sh2] Shokurov V. V. 3-fold log models, J. Math. Sci. 81 (1996), 2666-2699

[Sp] Springer T. Invariant theory, Lect. Notes. Math. 585, Springer, 1977.

[W] Weber, H.: Lehrbuch der Algebra. II. Chelsea: New York, 1961.

D. M.: Mathématiques - BÂt. M2, Université Lille 1, F-59655 Villeneuve d'Ascq Cedex, France

E-mail address: markushe@gat.univ-lille1.fr

Yu. G.: Algebra Section, Dept. of Mathematics, Moscow State University, 117234 Moscow, Russia

E-mail address: prokhoro@mech.math.msu.su, prokhoro@nw.math.msu.su, 